\title{
Principles, Techniques and Perspectives on Optimization and $\mathrm{HCl}$
}

\section{Per Ola Kristensson}

Department of Engineering

University of Cambridge

Cambridge, United Kingdom

Xiaojun Bi

Google, Inc.

Mountain View, California, USA

\section{Andrew Howes}

School of Computer Science

University of Birmingham

Birmingham, United Kingdom

Antti Oulasvirta

Department of Communications

and Networking

Aalto University

Helsinki, Finland

Permission to make digital or hard copies of part or all of this work for personal or classroom use is granted without fee provided that copies are not made or distributed for profit or commercial advantage and that copies bear this notice and the full citation on the first page. Copyrights for third-party components of this work must be honored. For all other uses, contact the owner/author(s). Copyright is held by the author/owner(s).

CHI'15 Extended Abstracts, Apr 18-23, 2015, Seoul, Republic of Korea. ACM 978-1-4503-3146-3/15/04

http://dx.doi.org/10.1145/2702613.2702662
Roderick Murray-Smith

of Computing Science

\section{Harold Thimbleby}

Department of Computer Science

School of Computing Science

versity of Glasgow

Shumin Zhai

Google, Inc.
Mountain View, California, USA

\begin{abstract}
We propose a workshop on the rapidly emerging topic of optimization and computational design in humancomputer interaction (HCI). The workshop will tackle the following perspectives: defining and eliciting optimality criteria, optimizing at scale, optimization and user models, optimization for safety, optimization and design practice, optimization and users' perception of performance, and critical perspectives.
\end{abstract}

\section{Author Keywords}

Optimization; modeling; inference; machine learning

\section{ACM Classification Keywords}

H.5.m. Information interfaces and presentation (e.g., HCI): Miscellaneous.

\section{Introduction}

Everyday we use optimized products and services, such as cars, thermostats, and public transportation systems. Indeed, in engineering and product design optimization is regarded a fundamental design principle $[8,13]$. It is therefore natural that optimization is also investigated within the context of human-computer interaction (HCI). The availability of big data, nexpensive sensors, and more computational processing power, in combination with recent dramatic progress in machine learning has led to 
reconsiderations of optimization and computational design approaches in $\mathrm{HCI}$. It is only recently that $\mathrm{HCI}$ researchers have begun to systematically explore stateof-the-art optimization approaches in $\mathrm{HCI}$ and this emerging research direction opens up many new perspectives that needs to be explored by a crossdisciplinary community of HCI scholars. The purpose of this workshop is to unify an emerging cross-disciplinary community of $\mathrm{HCI}$ researchers interested in understanding how optimization can further improve the design of new user interfaces.

\section{Perspectives on Optimization}

A principle underpinning this workshop is that optimization is a broad theme that overlaps with many areas of HCI. However, researchers are scattered across different subareas. This workshop serves to unify these research efforts by exploring the following perspectives on optimization, which also reflect the expertise of the co-organizers of the workshop.

Defining and Eliciting Optimality Criteria

Optimization nearly always relies on identifying objective optimality criteria, which can be difficult to define [7]. First, optimization often involves ill-posed inverse problems, which in practice often amounts to incorporating less than ideal regularization features into the cost functions to limit the complexity of the final solutions. An alternative approach would be to attempt to bias optimization toward certain classes of solutions. One recent example in $\mathrm{HCI}$ in this direction is keyboard layout optimization. Optimized layouts are usually very different from Qwerty, imposing large burdens in learning. Prior research [1,2] addressed this problem by introducing Qwerty constraints/similarity to bias the optimized layouts towards Qwerty.
A second issue when definition optimality criteria involves accommodating the diversity of user groups, such as older adults and users with disabilities, and use contexts, such as whether users are mobile, encumbered or surrounded by a crowd. How can we best elicit sound optimality criteria for different user groups and use contexts?

\section{Optimizing at Scale}

How does the availability of large amounts of data at high rates change the nature of optimization in interface design? Websites and other connected user interfaces such as mobile apps and wearable interfaces can provide data from potentially millions of users in a short time period. This can provide more evidence to improve a user interface than years of design trial and error. One recent example in $\mathrm{HCI}$ is the optimization of touch point detection by crowdsourcing [4].

Alternatively, simulations can be used to guide optimization and design. One recent approach has been explored in the area of brain-computer interaction (BCI), which investigated the relationship between simulation models in $\mathrm{BCI}$ and the involvement of endusers in the different stages of design of a BCI interface [10]. A better understanding of how we can best leverage large-scale data sources and simulations to explore a massive amount of design solutions can potentially require the involvement of both optimization experts and designers.

Optimization and User Models

Solving design optimization problems might be informed by models that predict user performance. It turns out that, while such models are difficult to build - because of the extraordinary flexibility of human cognition - recent developments in cognitive science 
have the potential to form the basis of a new approach. This approach relies on optimal control theory to derive user behavior from theories of underlying psychological constraints and subjective utility [9]. Optimal design might therefore be facilitated by optimal control theories of the user.

Another aspect is leveraging relationships between user models and optimization techniques. One recent example is the TrueSkill system, which optimizes the matching of evenly skilled participants in order to ensure an enjoyable game for all participants [5].

\section{Optimization for Safety}

Optimization and computational design can also be used to design for safety, for instance in the health care domain. This has for example been explored by using state machines and graph theory as a foundation to design better interactive systems and devices [12]

\section{Optimization and Design Practice}

Optimal parameters of design and even whole interface designs can be identified in very large, multidimensional design spaces. Interactive design tools using model-based optimization (e.g. [3]) allow designers to delegate well-known aspects of design problems to a computer. Exact approaches for optimization require using objective criteria for goal setting and assessment of progress. However, how this approach can become compatible with design practice is an open research question. While most previous work in optimization in HCI has focused on virtual keyboard design and intelligent systems, there is emerging interest in optimization and computational design in interaction techniques, web services, automotive UIs, programming environments, and continuous control. However, in order to successfully use optimization approaches to design products and services, we need to have a better understanding of how we can integrate optimization into design practice.

Optimization and Users' Perception of Performance An emerging research question is in $\mathrm{HCI}$ is the relationship between optimization for user performance and users' perception of their own performance [6]. Users may not always subjectively detect objective performance improvements. Increasing knowledge in this emerging area of research can better inform us when it is sensible to optimize and can also be used to balance trade-offs in design, for instance balancing efficiency with aesthetics.

\section{Critical Perspectives}

Should we optimize user interfaces? Which optimality criteria are worth considering? Do we want to optimize for speed or should we also consider slow change interaction design processes [11]? We welcome critical perspectives from all areas of $\mathrm{HCI}$ to discuss critical perspectives on optimization and $\mathrm{HCI}$.

\section{Workshop Goals}

Community Building

A major goal is to broaden the participation in the workshop, in particular from two directions. First, we plan to actively advertise the workshop to the wider engineering community, including researchers

investigating optimization techniques in engineering design, product design, machine learning and signal processing. Second, we will recruit participants from the wider $\mathrm{CHI}$ community to participate and provide critical perspectives on optimization and $\mathrm{HCI}$. 


\section{CHI Focus}

Given the cross-disciplinary nature of the community, researchers are spread across different conferences in HCI, such as UIST, EICS and IUI, and across different communities including engineering design and product design. A goal of this workshop is to advertise $\mathrm{CHI}$ as a natural and compelling center for research on optimization and $\mathrm{HCI}$.

\section{Research Dialogues}

Since researchers on optimization and $\mathrm{HCI}$ are active across the entire $\mathrm{HCI}$ spectrum, the scientific dialogue easily becomes unfocused and researchers investigating different perspectives on optimization and HCI may not always be aware of progress being made in other areas. A primary goal of this workshop is to bring all these researchers together to discuss difficult issues that are hard or near impossible to handle within the traditional format of research papers in conferences and journals.

\section{Conclusions}

Optimization and $\mathrm{HCI}$ is an emerging research area. However, currently our community is scattered across different research fields and areas of HCI. There are also a number of topics that are difficult to discuss in the traditional format of technical papers. This workshop serves to unify this diverse community and center it at $\mathrm{CHI}$. A further goal is to invite optimization researchers from engineering and product design and to explore crosscutting themes.

\section{References}

[1] Bi, X., Smith, B.A., and Zhai, S. 2010. QuasiQwerty soft keyboard optimization. In Proc. CHI (2010), 283-286.
[2] Dunlop, M.D. and Levine, J. 2012. Multidimensional Pareto optimization of touchscreen keyboards for speed, familiarity, and improved spell checking. In Proc. CHI (2012), 2669-2678.

[3] Gilles, B., Oulasvirta, A., Kötzing, T. and Hoppe, S. 2013. Menuoptimizer: interactive optimization of menu systems. In Proc. UIST (2013), 331-342.

[4] Henze, N., Rukzio, E. and Boll, S. 2012.

100,000,000 taps: analysis and improvement of touch performance in the large. In MobileHCI (2012), 133142.

[5] Herbrich, R., Minka, T. and Graepel, T. 2007. TrueSkill(TM): a Bayesian skill rating system. In NIPS (2007), 569-576.

[6] Nicosia, M., Oulasvirta, A. and Kristensson, P.O. 2014. Modeling the perception of user performance. In Proc. CHI (2014), 1747-1756.

[7] Nielsen, JB. 2014. Systems for Personalization of Hearing Instruments. PhD thesis, Technical University of Denmark.

[8] Pahl, G. and Beitz, W. 1996. Engineering Design A Systematic Approach. $2^{\text {nd }}$ Edition, Springer.

[9] Payne, S. J., \& Howes, A. (2013). Adaptive interaction: a utility maximization approach to understanding human interaction with

technology. Synthesis Lectures on Human-Centered Informatics 6(1): 1-111.

[10] Quek, M. 2013. The Role of Simulation in

Developing and Designing Applications for 2-Class Motor Imagery Brain-Computer Interfaces, PhD thesis, University of Glasgow.

[11] Siegel, M.A. and Beck, J. 2014. Slow change interaction design. Interactions 21(1): 28-35.

[12] Thimbleby, H. 2010. Press On: Principles of Interaction Programming. MIT Press.

[13] Ulrich, K.T. and Eppinger, S.D. 2008. Product Design and Development. $4^{\text {th }}$ Edition, McGraw-Hill. 